\title{
Distribution and evolution of galaxy groups in the Ursa Major supercluster
}

\author{
M. O. Krause' ${ }^{1}$, A. L. B. Ribeiro ${ }^{1}$, and P. A. A. Lopes $^{2}$ \\ ${ }^{1}$ Laboratório de Astrofísica Teórica e Observacional Departamento de Ciências Exatas e Tecnológicas Universidade Estadual de \\ Santa Cruz, 45650-000 Ilhéus-BA, Brazil \\ e-mail: krausefisico@ig.com.br; albr@uesc.br \\ 2 Observatório do Valongo, Universidade Federal do Rio de Janeiro, 20080-090 Rio de Janeiro-RJ, Brazil \\ e-mail: paal05@gmail.com
}

Received 20 July 2012 / Accepted 26 January 2013

ABSTRACT

\begin{abstract}
Context. We study an SDSS sample of galaxies within $\sim 50 \mathrm{Mpc}$ of the nominal center of the Ursa Major supercluster.
Aims. Our aim is to study galaxy distribution around groups in the supercluster and the link between the distribution of relaxed and nonrelaxed galaxy systems with respect to the supercluster environment.

Methods. Using the FoF algorithm, 40 galaxy groups were identified in this region. Velocity distributions for these groups were studied after applying a $3 \sigma$-clipping routine for outlier removal. We classified the systems according to the results of normality and substructure tests applied to member galaxies. Then, we studied the relative distribution of relaxed and nonrelaxed systems across the supercluster.

Results. We find that $68 \%$ of galaxy groups are Gaussian and that all the non-Gaussian systems have substructures and probably correspond to multimodal systems in redshift space. We also find that the Gaussian systems inhabit the denser regions of the supercluster, with higher densities of both red and blue galaxies within $2.5 \mathrm{~h}^{-1} \mathrm{Mpc}$, and have smaller group-group pairwise separations. Conclusions. Our results suggest a spatial segregation of dynamical states, where relaxed systems may have formed and evolved earlier and faster around high-density peaks, while nonrelaxed systems may be growing slower on the peripheries of lower density peaks. In this picture, galaxy clustering seems to be prompting a continuous internal evolution in the supercluster, with several groups collapsing into the more evolved and contracted regions.
\end{abstract}

Key words. galaxies: clusters: general - galaxies: groups: general

\section{Introduction}

Groups of galaxies contain most of galaxies in the Universe and are the link between individual galaxies and large-scale structures (e.g., Huchra \& Geller 1982; Geller \& Huchra 1983; Nolthenius \& White 1987; Ramella et al. 1989). The dissipationless evolution of these systems is dominated by gravity. Interactions over a relaxation time tend to distribute the velocities of the galaxy members into a Gaussian distribution (e.g. Bird \& Beers 1993). Although the theoretical line-of-sight (los) velocity distribution expected in galaxy systems is not exactly Gaussian (e.g. Merritt 1987; Kazantzidis et al. 2004), phenomenological evidence has been suggesting for a long time that normality can be assumed for systems in dynamical equilibrium (e.g. Yahil \& Vidal 1977).

Several works have shown that the analysis of velocity distributions can separate galaxy groups into relaxed or nonrelaxed systems (e.g. Hou et al. 2009; Ribeiro et al. 2010, 2011). Actually, several important properties of groups can be studied from this perspective. For instance, Hou et al. $(2009,2012)$ find rising velocity dispersion profiles for non-Gaussian (NG) groups. They have also shown that the majority of NG groups have substructures. Ribeiro et al. (2010) found that galaxies are redder and brighter in Gaussian (G) groups out to $4 R_{200}$. Martinez \& Zandivarez (2012) have studied the luminosity functions of $\mathrm{G}$ and $\mathrm{NG}$ groups, concluding that $\mathrm{G}$ groups have a brighter characteristic magnitude. Einasto et al. (2012a,b) studied velocity distributions to find signatures of multimodality in SDSS clusters.

The NG nature of the velocity distribution of NG groups must be caused by recent group-group mergers. At the same time, NG groups could be contaminated by chance projections. It is therefore important to establish the real nature of NG groups and how they are linked to the environment. We study an Sloan Digital Sky Survey (SDSS) sample of galaxies within $\sim 50 \mathrm{Mpc}$ of the nominal center of the Ursa Major supercluster (UMaS) aiming to establish a relation between large-scale environment and the evolution of galaxy groups. After identifying groups in the supercluster using the friends-of-friends $(\mathrm{FoF})$ algorithm, we split up the sample into relaxed and nonrelaxed systems according to the velocity distribution of member galaxies. Then, we investigate the distribution of galaxy groups in the supercluster with respect to their dynamical state. The paper is organized as follows. Section 2 presents the data and methods we have used, Sect. 3 presents our analysis, and Sect. 4 briefly summarizes the main conclusions of the work.

\section{Data}

In this work, we have used galaxies from the SDSS-DR6 (Adelman-McCarthy et al. 2008) within $\sim 50 \mathrm{Mpc}$ around the coordinates of the cluster Abell $1383\left(=177.46^{\circ}\right.$, Dec $=54.62^{\circ}$, 
and $z=0.059$ ) taken here as the nominal (and arbitrary) "center" of the UMaS. The redshifts are in the range $0.045<z<0.075$. We have found 18204 galaxies in this region with apparent magnitude limit $m_{\mathrm{r}}=17.77$. Clusters were identified using the FoF algorithm (Huchra \& Geller 1982), following the choice of linking lengths by Berlind et al. (2006) $\left(b_{\perp}=0.14\right.$ and $b_{\|}=$ $0.75)^{1}$, which were optimized for SDSS. The FoF algorithm with these linking lengths finds galaxy groups with $N_{\text {gal }} \geq 10$ that have an unbiased multiplicity function (see Berlind et al. 2006). These settings led us to find 40 galaxy groups in the UMaS, with numbers of galaxies in the range $15 \leq N_{\text {gal }} \leq 94$. Our catalog contains all but one of the 23 galaxy systems known in the region $^{2}$ (see Kopylova \& Kopylov 2009).

Velocity distributions for the identified groups were studied after applying a $3 \sigma$ clipping routine for removing outliers. First, a virial analysis ${ }^{3}$ is done following Carlberg et al. (1997), where $R_{200}=\sqrt{3} \sigma /[10 H(z)]$ and $M_{200}=3 R_{200} \sigma^{2} / G$. Then, we applied three well known normality tests to the cleaned velocity distributions - the Anderson-Darling (AD), Shapiro-Wilk (SW) and Jarque-Bera (JB) tests - to decide if a galaxy system is Gaussian or NG. Our criterium is that at least two tests should reject normality at the $95 \%$ confidence level to be considered NG; if not, it will be considered Gaussian. Finally, we applied two substructure tests - the $\beta$ (West et al. 1988) and $\Delta$ tests (Dressler $\&$ Schectman 1988) - to decide if a galaxy system has substructures. Our criterion is that a galaxy system is considered to have substructures if at least one of the two tests rejects regularity with $95 \%$ confidence level. Results are presented in Table 1, where columns are (1) group identification; (2) and (3) equatorial coordinates in degrees; (4) average redshift of members after removing outliers; (5) velocity dispersion in $\mathrm{km} \mathrm{s}^{-1}$; (6) $R_{200}$ radius in $h^{-1} \mathrm{Mpc}$; (7) logarithm of $M_{200}$ in $h^{-1} M_{\odot} ;(8)$ total number of galaxies after outlier removal; (9) results for the NG nature of the los velocity distribution: 0 for Gaussian and 1 for NG distributions; (10) results for substructure tests: 0 for regular and 1 for irregular systems; (11) Abell cluster identification.

\section{Analysis}

\subsection{Multimodality}

We find that 27 out of 40, i.e. $68 \%$, of galaxy groups are Gaussian and that all the NG systems have substructures. Also, we find that four groups have substructures (as indicated by the $\beta$ test) but have Gaussian velocity distributions according to our criteria. If we include them as nonrelaxed systems, the total fraction of virialized groups would fall to $58 \%$. We take a conservative view to define nonrelaxed groups and assume that substructures are dynamically significant only if they are also associated to non-Gaussianities in the velocity distribution of a galaxy system.

Following Ribeiro et al. (2011), we applied the KolmogorovSmirnov (KS) and the Cramér-von Mises (CvM) two-sample tests on the G and NG subsamples. We find that NG velocity groups are signifcantly larger and more massive at the $95 \%$ confidence level for both tests, with best fit relations:

$$
\begin{aligned}
& -\bar{R}_{200}^{\mathrm{NG}} \simeq(1.27 \pm 0.22) \bar{R}_{200}^{\mathrm{G}} \\
& -\bar{M}_{200}^{\mathrm{NG}} \simeq(2.00 \pm 0.65) \bar{M}_{200}^{\mathrm{G}}
\end{aligned}
$$

\footnotetext{
1 The parameters $b_{\perp}$ and $b_{\|}$are the projected and line-of-sight linking lengths in units of the mean intergalaxy separation.

2 The missing cluster is beyond our search radius.

3 In this work, cosmology is defined by $\Omega_{\mathrm{m}}=0.3, \Omega_{\lambda}=0.7$, and $H_{0}=$ $100 h \mathrm{~km} \mathrm{~s}^{-1} \mathrm{Mpc}^{-1}$.
}

\begin{tabular}{|c|c|c|c|c|c|c|c|c|c|}
\hline$\#$ & $\begin{array}{l}\text { RA } \\
\left({ }^{\circ}\right)\end{array}$ & $\begin{array}{l}\text { Dec } \\
\left({ }^{\circ}\right)\end{array}$ & $\bar{z}$ & $\begin{array}{c}\sigma \\
\left(\mathrm{km} \mathrm{s}^{-1}\right)\end{array}$ & $\begin{array}{c}R_{200} \\
\left(h^{-1} \mathrm{Mpc}\right)\end{array}$ & $\begin{array}{l}\log M_{200} \\
\left(h^{-1} M_{\odot}\right)\end{array}$ & $N_{\text {gal }}$ & $V S$ & Abell \\
\hline$\overline{1}$ & 197.07 & 39.83 & 0.0705 & 342 & 0.57 & 13.67 & 50 & 11 & - \\
\hline 2 & 178.32 & 44.10 & 0.0707 & 250 & 0.42 & 13.26 & 33 & 00 & - \\
\hline 3 & 172.31 & 56.28 & 0.0573 & 252 & 0.42 & 13.27 & 26 & 11 & A1291 \\
\hline 4 & 176.69 & 55.77 & 0.0785 & 387 & 0.64 & 13.83 & 94 & 11 & 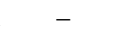 \\
\hline 5 & 180.98 & 52.41 & 0.0624 & 181 & 0.30 & 12.84 & 25 & $\begin{array}{ll}0 & 0\end{array}$ & A145 \\
\hline 6 & 156.56 & 47.82 & 0.0620 & 282 & 0.47 & 13.42 & 67 & 11 & A1003 \\
\hline 7 & 183.54 & 60.00 & 0.0602 & 212 & 0.36 & 13.05 & 34 & $\begin{array}{ll}0 & 0\end{array}$ & A1507 \\
\hline 8 & 174.76 & 57.45 & 0.0665 & 190 & 0.32 & 12.90 & 21 & $\begin{array}{ll}0 & 0\end{array}$ & A1318 \\
\hline 9 & 149.66 & 53.86 & 0.0470 & 272 & 0.46 & 13.38 & 36 & 11 & - \\
\hline 10 & 176.75 & 55.69 & 0.0514 & 233 & 0.39 & 13.17 & 33 & $\begin{array}{ll}0 & 0\end{array}$ & A1377 \\
\hline 11 & 157.64 & 53.33 & 0.0643 & 232 & 0.39 & 13.17 & 30 & 01 & - \\
\hline 12 & 168.39 & 54.79 & 0.0710 & 193 & 0.32 & 12.92 & 29 & $\begin{array}{ll}0 & 0\end{array}$ & - \\
\hline 13 & 168.49 & 57.03 & 0.0474 & 255 & 0.43 & 13.29 & 39 & $\begin{array}{ll}0 & 0\end{array}$ & - \\
\hline 14 & 181.69 & 56.93 & 0.0644 & 206 & 0.35 & 13.01 & 30 & $\begin{array}{ll}0 & 0\end{array}$ & A1436 \\
\hline 15 & 171.99 & 67.09 & 0.0549 & 32 & 0.55 & 3.61 & 56 & $\begin{array}{ll}0 & 1\end{array}$ & A1279 \\
\hline 16 & 197.27 & 54.60 & 0.0642 & 22 & 0.37 & 3.10 & 21 & 00 & - \\
\hline 17 & 182.60 & 45.44 & 0.0660 & 20 & 0.33 & 2.97 & 36 & $\begin{array}{ll}0 & 0\end{array}$ & - \\
\hline 18 & 161.10 & 38.14 & 0.0428 & 33 & 0.57 & 13.66 & 55 & 11 & - \\
\hline 19 & 195.58 & 60.72 & 0.0702 & 27 & 0.46 & 3.38 & 40 & 11 & - \\
\hline 20 & 181.29 & 54.42 & 0.0503 & 23 & 0.39 & 13.17 & 28 & $\begin{array}{ll}0 & 1\end{array}$ & - \\
\hline 21 & 168.63 & 49.42 & 0.0733 & 259 & 0.43 & 3.31 & 39 & 00 & - \\
\hline 22 & 177.15 & 54.66 & 0.0596 & 207 & 0.35 & 13.02 & 27 & 11 & A1383 \\
\hline 23 & 172.46 & 36.36 & 0.0625 & 275 & 0.46 & 3.39 & 35 & $\begin{array}{ll}0 & 0\end{array}$ & - \\
\hline 24 & 180.45 & 38.30 & 0.0646 & 20 & 0.35 & 3.02 & 21 & $\begin{array}{ll}0 & 0\end{array}$ & - \\
\hline 2 & 1 & 45 & 0 & 336 & 0.57 & 3.65 & 49 & 11 & - \\
\hline 26 & 181.58 & 42.69 & 0.0526 & 282 & 0.48 & 13.42 & 35 & 11 & A 1461 \\
\hline 27 & 178 & 49 & 0.0541 & 25 & 0.43 & 3.30 & 53 & $\begin{array}{ll}0 & 0\end{array}$ & - \\
\hline 28 & 168.14 & 41.07 & 0.0728 & 287 & 0.48 & 13.44 & 41 & 11 & - \\
\hline 29 & 189.56 & 42.00 & 0.0656 & 206 & 0.35 & 13.01 & 21 & $\begin{array}{ll}0 & 0\end{array}$ & - \\
\hline 30 & 184.97 & 59.79 & 0.0444 & 331 & 0.56 & 13.63 & 55 & 11 & $1 \quad$ A1534 \\
\hline 31 & 160.71 & 45.22 & 0.0493 & 29 & 0.50 & 13.49 & 51 & 00 & - \\
\hline 32 & 170.37 & 47.03 & 0.0534 & 18 & 0.31 & 12.85 & 18 & 01 & - \\
\hline 33 & 159.19 & 56.65 & 0.0458 & 308 & 0.52 & 13.54 & 58 & 00 & - \\
\hline 34 & 202.42 & 44.78 & 0.0613 & 251 & 0.42 & 13.27 & 44 & 11 & - \\
\hline 35 & 161.15 & 57.57 & 0.0732 & 264 & 0.44 & 13.33 & 40 & 00 & - \\
\hline 36 & 197.49 & 49.56 & 0.0554 & 237 & 0.40 & 13.19 & 35 & 0 & - \\
\hline 37 & 170.34 & 41.94 & 0.0601 & 151 & 0.25 & 12.61 & 15 & 0 & - \\
\hline 38 & 203.11 & 57.41 & 0.0528 & 281 & 0.47 & 13.42 & 41 & 0 & - \\
\hline 39 & 172.47 & 54.30 & 0.0693 & 215 & 0.36 & 13.07 & 33 & 00 & $0 \quad$ A1270 \\
\hline 40 & 166.68 & 43.83 & 0.0583 & 221 & 0.37 & 13.10 & 32 & 00 & $\begin{array}{l}0 \\
\text { A1169 }\end{array}$ \\
\hline
\end{tabular}

Table 1. Properties of 40 galaxy groups identified in the UMaS.

Notes. Columns 9 and 10 indicate results for normality and substructure tests described in the text.

with $[(p=0.0065-\mathrm{KS}),(p=0.0019-\mathrm{CvM})]$ for the radius distributions, and $[(p=0.0029-\mathrm{KS})(p=0.0017-\mathrm{CvM})]$ for the mass distributions. This result is similar to the one found by Ribeiro et al. (2011), a possible consequence of applying the virial analysis to nonvirialized objects. Actually, we can assume the velocity distributions of NG groups as Gaussian mixtures with unknown number of components, and use the Dirichlet process mixture (DPM) model to study the velocity distributions (see Ribeiro et al. 2011 for details). The number of components in the mixture is computed using the $\mathrm{R}$ language and environment (R Develoment Core Team) under the dpmixsim library (da Silva 2009). We find the following results:

$$
\begin{aligned}
& \text { - Unimodal groups: } 3,9,34 \\
& \text { - Bimodal groups: 4, 6, 18, 19, 22, 25, 26, 28, } 30 \\
& \text { - Trimodal group: } 1 \text {. }
\end{aligned}
$$

In Fig. 1 we plot the velocity distributions for typical cases of NG groups. Multimodality seems to be the prominent cause of non-Gaussianity in galaxy group velocity distributions, corresponding to 10 out of 13 , i.e. $77 \%$ of the NG cases in the UMaS. 
M. O. Krause et al.: Distribution and evolution of galaxy groups in the UMaS
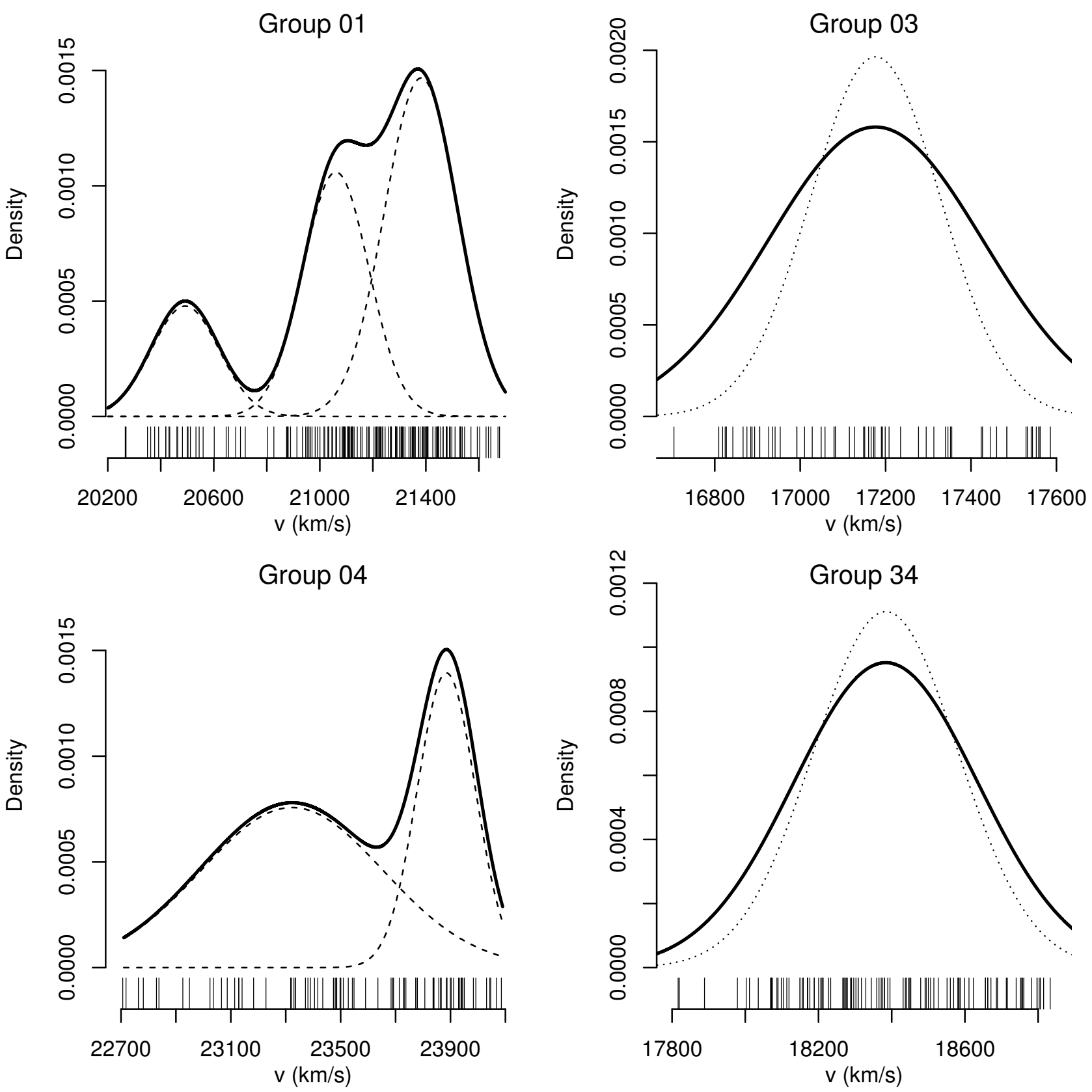

Fig. 1. Four examples of multimodality diagnostics for groups in the UMaS region. Vertical bars depict individual galaxies in each group. The solid lines indicate the best Gaussian mixture for for radial velocities distributions in $\mathrm{km} \mathrm{s}^{-1}$. The dashed lines show the individual components of the multimodal systems. The dotted lines indicate the average Gaussian curve for 1000 resamplings of groups 03 and 34, unimodal but non-Gaussian systems.

There are three unimodal systems $(\# 03, \# 09$, and \#34) all having negative kurtosis excess $(k=-1.26,-0.87$, and $k=-1.05$, respectively) - the mean values for Gaussian and NG groups are $k_{\mathrm{G}}=-0.34$ and $k_{\mathrm{NG}}=-1.16$ A negative kurtosis corresponds to a flattened and usually multimodal distribution. However, if the separation between the modes is too small, the DPM model is unable to recognize distinct components. In Fig. 1, we illustrate two cases of NG unimodal systems, also plotting the Gaussian curve resulting from 1000 resamplings keeping $90 \%$ of the data ${ }^{4}$. For each group we apply the normality tests mentioned in Sect. 2 to the new velocity distribution.

\footnotetext{
4 For instance, group \#03 has 26 members, thus each resampling provides a reduced group with 23 members.
}

The reduced samples classified as normal distributions are used to compute the average Gaussian we plot in Fig. 1.

\subsection{Supercluster environment}

Now, we try to link the dynamical state of galaxy systems and the supercluster environment. The spatial distribution of galaxy groups in the UMaS region is presented in Fig. 2 in supergalactic Cartesian coordinates ${ }^{5}$, where we see galaxies and groups.

5 The supergalactic coordinates are based on the preferred plane of the Local Supercluster. After the coordinate transformation, the Abell cluster A1383 was chosen to be at the center of galaxy distribution. 


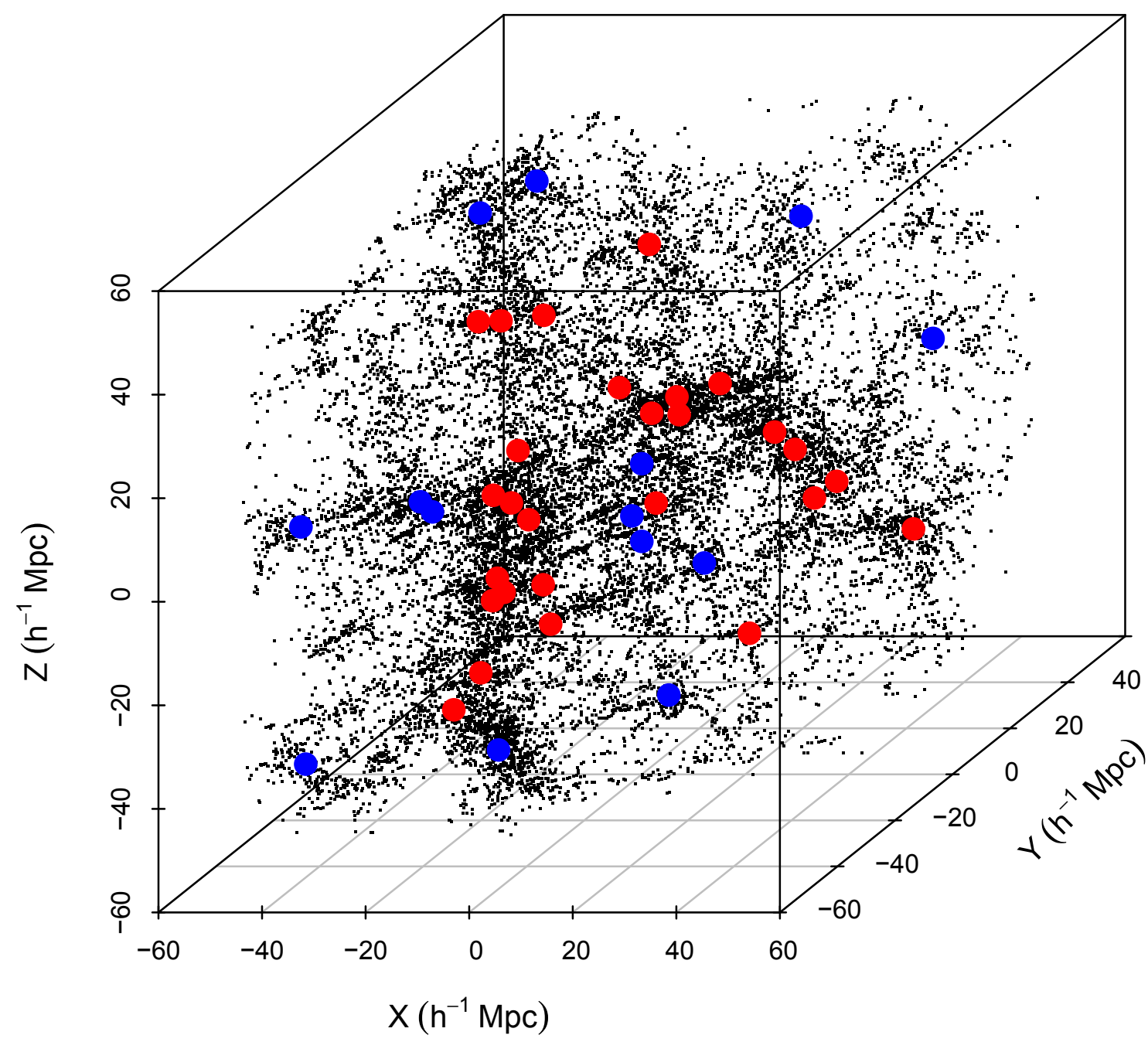

Fig. 2. Distribution of galaxies and galaxy systems in the UMaS in supergalactic coordinates. Galaxies are in black; Gaussian groups and nonGaussian are in red and blue, respectively.

From this figure, it seems that $\mathrm{G}$ groups inhabit the denser regions of the supercluster, approximately following the central arch-shaped structure in the plot. That could be hinting at some environmental influence in the galaxy groups spatial distribution.

We have used two simple indicators to probe the possible relation between galaxy groups and the supercluster environment.

\subsubsection{Distribution of galaxies around groups}

First, we built the stacked G and NG galaxy number density (in redshift space) up to $2.5 h^{-1} \mathrm{Mpc}$, around the groups' centers, a distance corresponding to $\simeq 6 \bar{R}_{200}$ for our sample. Results are presented in Fig. 3, where we see similar shapes for the profiles around $\mathrm{G}$ and $\mathrm{NG}$ groups, but with densities systematically higher in the case of $\mathrm{G}$ groups. Error bars in this plot are obtained from a bootstrap technique with 1000 resamplings applied to the galaxy groups. This result indicates that $\mathrm{G}$ systems present significantly richer surroundings than NG sytems, with ratio of number densities of galaxies around $\mathrm{G}$ and $\mathrm{NG}$ groups following $\log \left[v_{\mathrm{NG}}(r) / v_{\mathrm{G}}(r)\right] \simeq 0.35 \pm 0.10(v$ is the galaxy number density).

We also classified galaxies into blue or red objects following the criterion of Strateva et al. (2001) - a red galaxy should have $u-r \geq 2.22$. This enables us to rebuild the galaxy density profiles for these two populations. The results for red galaxies are also presented in Fig. 3 (the middle plot), where we see that $\mathrm{G}$ groups have more red galaxies within $2.5 \mathrm{~h}^{-1} \mathrm{Mpc}$ than NG groups $\log \left[v_{\mathrm{NG}}^{\mathrm{r}}(r) / v_{\mathrm{G}}^{\mathrm{r}}(r)\right] \simeq 0.46 \pm 0.11$, but note that the difference are smaller at larger radii, reflecting (i) the well known correlation between colors and environment - the fraction of red galaxies decreases toward the outer part of clusters (e.g. Balogh et al. 2000; De Propis et al. 2004); and (ii) the quicker decline for $\mathrm{G}$ systems. At the same time, for blue galaxies the difference between $\mathrm{G}$ and $\mathrm{NG}$ systems is smaller, but still statistically significant: $\log \left[v_{\mathrm{NG}}^{\mathrm{b}}(r) / v_{\mathrm{G}}^{\mathrm{b}}(r)\right] \simeq 0.69 \pm 0.12$, see Fig. 3 (on the bottom plot). These results show that $\mathrm{G}$ groups are denser and redder than NG groups up to $2.5 h^{-1} \mathrm{Mpc}$.

\subsubsection{Distribution of groups through the UMaS}

Also, we studied the relative separation between groups in both $\mathrm{G}$ and $\mathrm{NG}$ subsets. We computed the distance from each group to the first $(k=1)$, second $(k=2)$, and third $(k=3)$ nearest groups in the spatial distribution. In Fig. 4, we illustrate our results for the supergalactic $Y Z$ projection ${ }^{6}$. The mean pairwise distances of

6 We found approximately the same results for the $X Y$ and $X Z$ projections. 


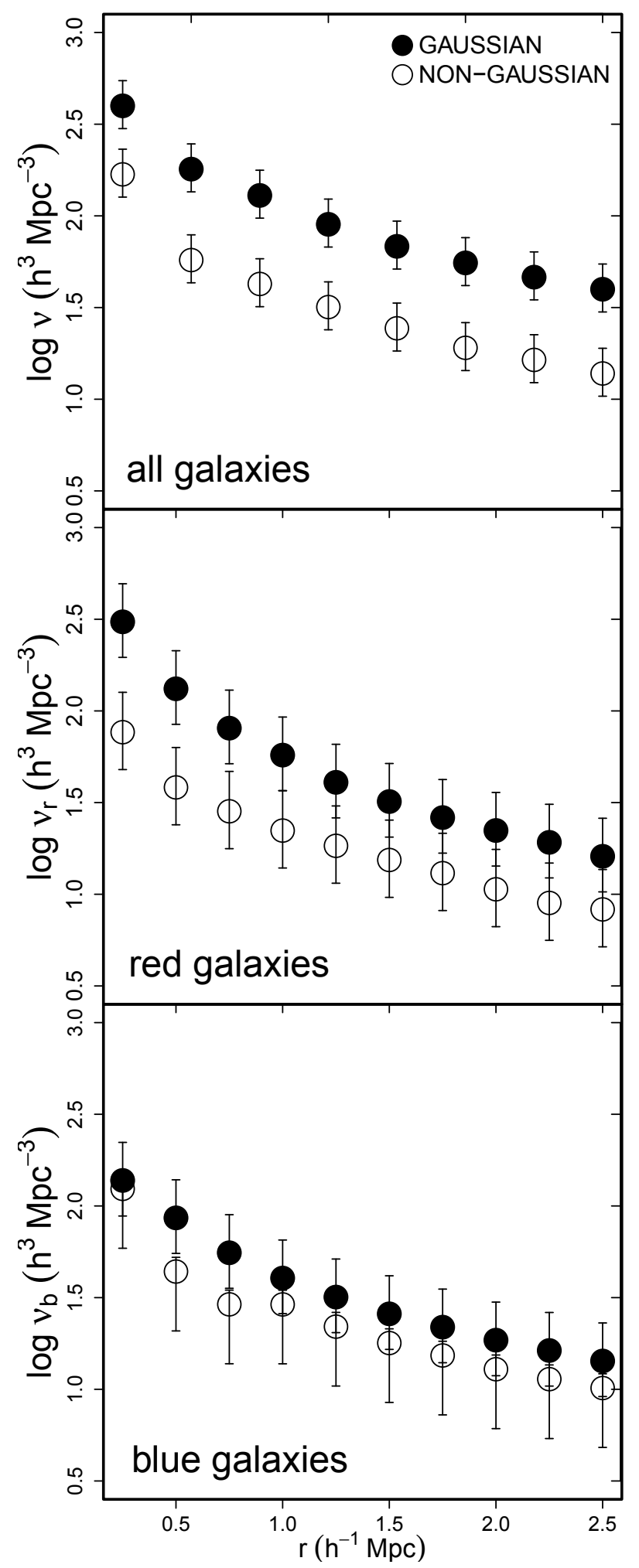

Fig. 3. Galaxy number density profiles in redshift space. Filled and open circles denote $\mathrm{G}$ and NG systems, respectively. Error bars are obtained from 1000 bootstrap resamplings of galaxy groups. Top, middle and bottom plots refer to all, red, and blue galaxies, respectively.

G groups show just a small variation with $k: 11 h^{-1} \mathrm{Mpc}(k=1)$, $12 h^{-1} \operatorname{Mpc}(k=2)$, and $14 h^{-1} \operatorname{Mpc}(k=3)$; while we see a wider variation for NG groups: $10 h^{-1} \mathrm{Mpc}(k=1), 19 h^{-1} \mathrm{Mpc}$ $(k=2)$, and $26 h^{-1} \mathrm{Mpc}(k=3)$. This suggests that $\mathrm{G}$ groups are closer to each other than NG groups.
We estimated the significance of the pairwise separation test by scrambling the identities of the $\mathrm{G}$ and $\mathrm{NG}$ groups 10000 times, preserving the overall numbers of 27 Gaussian and 13 non-Gaussian. We calculated the number of replicas with more extreme statistics than the input data (i.e. larger differences between $\mathrm{G}$ and NG mean pairwise separations). For $k=1$, we found 4356 cases, indicating that $\mathrm{G}$ and $\mathrm{NG}$ systems have the same distributions with respect to the first neighbor. However, for $k=2$ and $k=3$ we found 279 and 232 cases of more extreme statistics. We can therefore reject the null hypothesis of the same spatial distribution for G and NG systems at the $\sim 2.8 \%$ and $\sim 2.3 \%$ signficance levels, respectively. The difference between the spatial distributions of $\mathrm{G}$ and $\mathrm{NG}$ groups is reinforced by the result of the two-dimensional KS test (e.g. Press et al. 1992; Fasano \& Franceschini 1987), which evaluates the null hypothesis that a pair of data sets are drawn from the same distribution. We found that this is rejected at the $95 \%$ confidence level for all 2D data projections.

Combined, these results indicate that $\mathrm{G}$ groups probably inhabit denser regions, with higher densities of red galaxies, and are closer to each other (at least for $k=2$ and $k=3$ ) than NG groups - a trend that suggests an important environmental effect on structure formation through the UMaS volume.

\section{Discussion}

Superclusters are the largest structures identified in the universe. They correspond to complex systems comprising a few up to several galaxy clusters and groups experiencing long-term gravitational evolution. In this work, we studied a galaxy sample $\sim 50 \mathrm{Mpc}$ around an arbitrary center (the Abell cluster 1383) of the UMaS. We have addressed two questions: galaxy distribution around groups in the supercluster and the link between the distribution of relaxed and nonrelaxed galaxy systems with respect to the large-scale environment in which they are embedded. We find that $68 \%$ of galaxy groups are Gaussian and that all the NG systems have substructures. This corresponds to a lower fraction of relaxed groups in comparison to our previous studies (Ribeiro et al. 2010, 2011) ${ }^{7}$. This result cannot be due to the different criteria employed to split up G and NG groups, since we are being more conservative to define nonrelaxed systems in the present work. Alternatively, this may result from the supercluster environment, which could be favoring interactions and mergers between the groups. Actually, finding multimodality as the main cause of non-Gaussinity seems consistent with the idea of ongoing processes in nonrelaxed systems (e.g. Ribeiro et al. 2011; Hou et al. 2012; Einasto et al. 2012a).

In this respect, an important issue relates to the possibility of also having NG groups as the result of spurious members in the velocity distributions. Wojtak et al. (2007) show that most statistical methods (including the $3 \sigma$-clipping procedure used in the present work) exclude $60 \%-70 \%$ of unbound galaxies on average. The probability of chance alignments with the remaining interlopers was estimated with the following procedure:

1. We assume that all groups are $100 \%$ reliable in the membership.

2. We place all galaxies within them at the center, making their velocity dispersions nil.

3. And we rotate the cube around a random axis.

4. Then, we disperse the los velocities according to a Gaussian distribution for each group with the original velocity dispersion for the group.

7 But consistent with Hou et al. (2009) for CNOC2 data. 

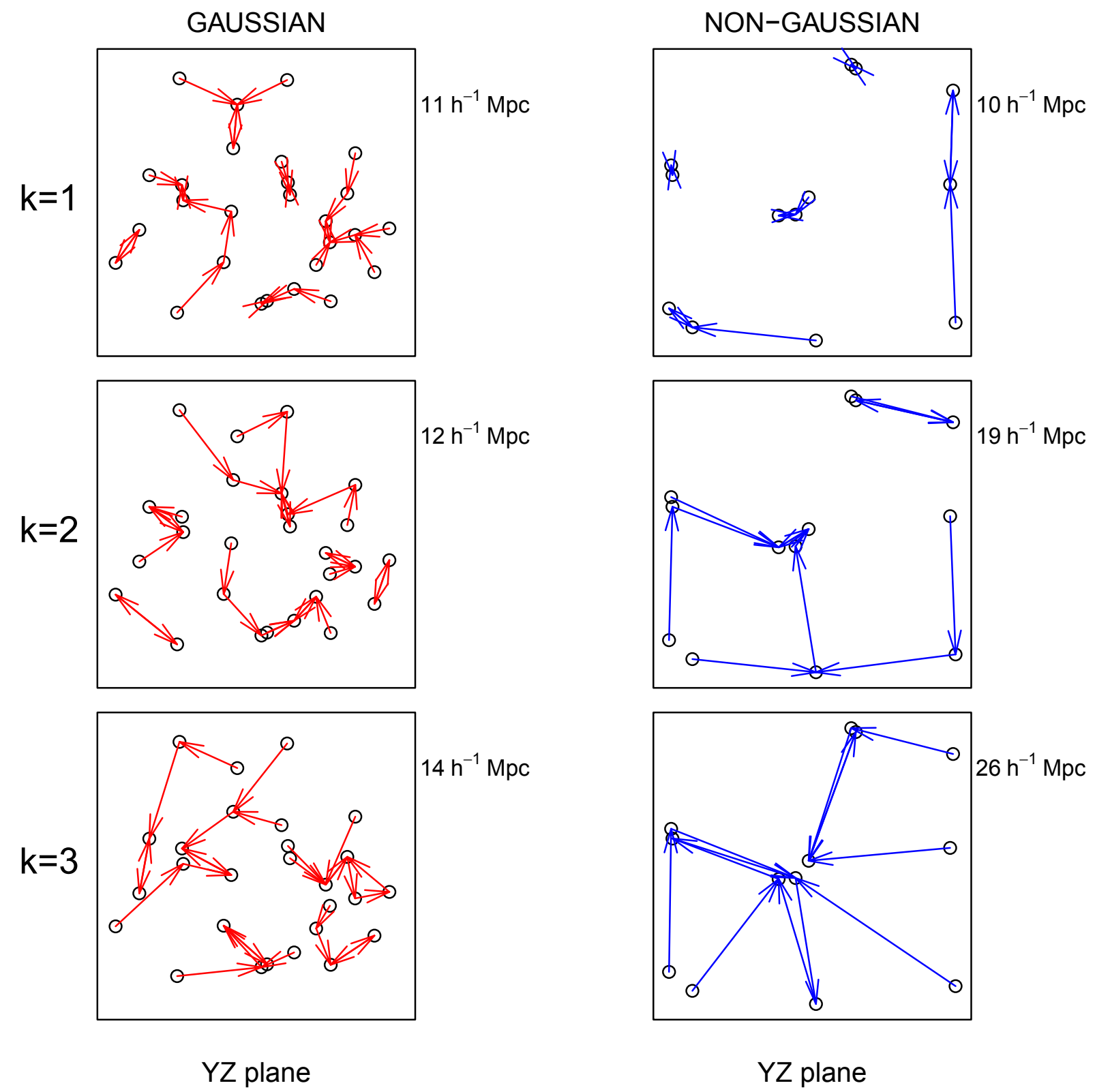

\section{YZ plane}

Fig. 4. Pairwise distances from each group to the first $(k=1)$, second $(k=2)$ and third $(k=3)$ nearest neighbors in the supergalactic $Y Z$ projection. The arrows point to the $k$ th nearest neighbor among groups of the same class. The red and blue arrows indicate distances for G and NG systems, respectively. The mean distance between groups appears on the right side of each box. The size of all boxes is $100 \times 100 \mathrm{~h}^{-1} \mathrm{Mpc}$.

5. Finally, we apply an FoF code with the same parameters mentioned in Sect. 2, and analyze the Gaussianity of the resulting groups.

After 1000 repetitions of this procedure, we found that $\sim 15 \%$ of the mock groups, which are Gaussian by construction, become NG, once additional members are included. This means that about two NG groups in our sample would correspond to Gaussian systems with spurious members in their velocity distributions. This fraction is too low to signicantly modify the results we found in the previous sections, so most of NG groups are probably portraits of multimodal systems.

Even presuming this is so, Gaussian groups are still dominant across the supercluster, which suggests the system is not young enough. Actually, Einasto et al. (2012b) show that superclusters have two main morphological types, spiders and filaments. Clusters in superclusters of spider morphology have higher probabilities to have substructure and higher peculiar velocities of their main galaxies than clusters in superclusters of filament morphology. The UMaS is classified as filament-type in that work, which suggests it might be dynamically evolved. In the same direction, we found that $G$ groups inhabit denser regions, with higher densities of both red and blue galaxies within $2.5 h^{-1} \mathrm{Mpc}$ and are closer to each other than NG groups, suggesting that relaxed systems may have formed and evolved earlier around high-density peaks, while nonrelaxed systems may be growing more slowly in the peripheries of lower density peaks. This shows the complex nature of superclusters so that further studies are needed to understand the role of the largescale environment in galaxy group distribution and evolution.

Acknowledgements. We thank the referee Gary Mamon for very useful suggestions. We are thankful to Jason Pinkney for making the substructure codes available. A.L.B.R. acknowledges the support of the CNPq, grants 306870/2010-0 and 478753/2010-1. P.A.A.L. acknowledges the support of the FAPERJ, grant E-26/110.237/2010, and CNPQ, grant 304692/2011-5. 
M. O. Krause et al.: Distribution and evolution of galaxy groups in the UMaS

\section{References}

Adelman-McCarthy, J. K., Adelman-McCarthy, J. K., Agüeros, M. A., et al. 2008, ApJS, 175, 297

Araya-Melo, P. A., Reisenegger, A., Meza, A., et al. 2009, MNRAS, 399, 97

Balogh, M., Navarro, J., \& Morris, S. L. 2000, ApJ, 540, 113

Berlind, A. A., Frieman, J., Weinberg, D. H., et al. 2006, ApJS, 167, 1

Bird, C., \& Beers, T. 1993, AJ, 105, 1596

Carlberg, R. G., Yee, H. K. C., \& Ellingson, E. 1997, ApJ, 478, 462

da Silva, A. F. 2009, Comput. Methods Programs Biomed., 94, 1

De Propis, R., De Propris, R., Colless, M., et al. 2004, MNRAS, 351, 125

Dressler, A., \& Shectman, S. A. 1988, AJ, 95, 985

Dünner, R., Araya, P. A., Meza, A., \& Reisenegger, A. 2006, MNRAS, 366, 803

Einasto, M., Vennik, J., Nurmi, P., et al. 2012a, A\&A, 540, A123

Einasto, M., Liivamägi, L. J., Tempel, E., et al. 2012b, A\&A, 542, A36

Fasano, G., \& Franceschini, A. 1987, MNRAS, 225, 155

Geller, M. J., \& Huchra, J. P. 1983, ApJS, 52, 61
Hou, A., Parker, L., Harris, W., \& Wilman, D. J. 2009, ApJ, 702, 1199 Hou, A., Parker, L. C., Wilman, D. J., et al. 2012, MNRAS, 421, 3594

Huchra, J. P., \& Geller, M. J. 1982, ApJ, 257, 423

Kazantzidis, S., Magorrian, J., \& Moore, B. 2004, ApJ, 601, 37

Merritt, D. 1987, ApJ, 313, 121

Kopylova, F. G., \& Kopylov, A. I. 2009, Astrophysical Bulletin, 64,

Martínez, H. J., \& Zandivarez, A. 2012, MNRAS, 419, L24

Nolthenius, R., \& White, S. D. M. 1987, MNRAS, 225, 505

Press, W. H., Teukolsky, S. A., Vetterling, W. T., \& Flannery, B. P. 1992,

Numerical Recipes in C, 2nd edn. (Cambridge: Cambridge University Press) Ramella, M., Geller, M., \& Huchra, J. P. 1989, ApJ, 344, 57

Ribeiro, A. L. B., Lopes, P. A. A., \& Trevisan, M. 2010, MNRAS, 409, L124

Ribeiro, A. L. B., Lopes, P. A. A., \& Trevisan, M. 2011, MNRAS, 413, L81

Strateva I., Ivezić, Ž., Knapp, G. R., et al. 2001, AJ, 122, 1861

West, M., Oemler, A., \& Dekel, A. 1988, ApJ, 327, 1

Wojtak, R., Lokas, E. L., Mamon, G. A., et al. 2007, A\&A, 466, 437

Yahil, A., \& Vidal, N. V. 1977, ApJ, 214, 347 